\title{
Neck Dissection Complications: Chylous Fistula and a New Conservative Management
}

\author{
Raffaele Rauso*1, Gian Paolo Tartaro ${ }^{2}$, Fabrizio Chirico ${ }^{3}$, Luigi Rugge ${ }^{3}$, Giada Albani ${ }^{3}$ and Giuseppe Colella ${ }^{1}$ \\ ${ }^{1}$ Professor in Maxillo-Facial Surgery, University of Campania Luigi Vanvitelli, Naples, Italy \\ ${ }^{2}$ Head of Maxillo-Facial Department, University of Campania Luigi Vanvitelli, Naples, Italy \\ ${ }^{3}$ Resident in Maxillo-Facial Surgery, University Federico II, Italy
}

Received: 盋: September 30, 2018; Published: 眥: October 09, 2018

*Corresponding author: Raffaele Rauso, Professor in Maxillo-Facial Surgery, University of Campania Luigi Vanvitelli, Naples, Italy

\begin{abstract}
Lymphatic fistula is one of the possible complication of neck dissection and may be very challenging to manage because of the anatomical complexities such as proximity to major vessels and variations. An untreated chyle fistula is a potentially dangerous condition: if not promptly identified and appropriately treated it could carry potentially to serious consequences as hypovolemia, hyponatremia, hypochloremia, hypoproteinemia and lymphopenia. Despite technological advances and new approaches described in the recent literature, there is still no clear treatment algorithm for this complication. Functional repair of the lymphatic duct injury should be the preferred solution, rather than an approach that obliterates the thoracic duct or chylous pathways, as this can have unwanted consequences such as redistribution of flow to produce distal complications. In chyle fistula following neck dissection for squamous tongue carcinoma, in case of impossibility of surgical approach due to patient general condition , fistula was successfully treated conservatively: TPN , octreotide continuous intravenous infusion associated to empiric procedure of sclerosant treatment through by washing of atossisclerol solution. The injection of this sclerosant agent into supraclavicular wound bed through the drainage tube determined rapid decline in fistula output. The treatment adopted was a new protocol of conservative management, able of inducing the healing of the fistula.
\end{abstract}

Abbreviations: LFD: low-Fat Diet; FFD: Fat-Free Diet; EFAs: Essential Fatty Acids; MCTs: Medium-Chain Triglycerides; TPN: Toatal Parenteral Nutrition

\section{Introduction}

Neck dissection, surgical procedure for removal of neck node cancer, has a low morbidity and mortality rates; however, the association of previous radiation therapy, poor general health, chronic malnutrition, alcoholism, diabetes mellitus, advanced age, and systemic illness, markedly increases the rate of complications [1]. Neck dissection post-operative complications include hematoma, wound infection, skin flap loss, salivary fistula, facial edema, electrolyte disturbances, carotid artery rupture and chylous fistula. Chyle can be identified by the appearance of a milky clouded fluid in drains and its accumulation can cause redness and swelling of the surrounding tissues. It is a rare complication occurring approximately in the $1-2 \%$ of patients who undergo neck dissection procedures: if untreated it could be a potentially dangerous condition that may lead to hypovolemia, hyponatremia, hypochloremia, hypoproteinemia and lymphopenia [2].

Factors favoring the occurrence of this injury are the anatomical variations in the terminal portion of the progress of the thoracic duct and the drain mode of injuried neck. In neck dissection, many lymphatic pathways are interrupted and the lymphatic flow towards the intact lymph collectors resumes tardive; usually the phenomenon of the local accumulation of chemical material in chylous fistula is progressively reduced in a few days. The amount of material collected by the drainage positioned during the surgical procedure, its persistence and composition guide diagnostic and therapeutic procedure [3]. The leak, if minimal, is usually controlled by aspiration, pressure dressings, and a low-fat diet. The occurrence of a massive lymphatic flow lead us to continue in research of new conservative treatment: even if surgical procedures, such as ligation of the offending thoracic duct, are indicated when the leak is extensive with more than $500 \mathrm{~mL}$ of drainage, because of the debilitated systemic condition of the patient that lead to the impossibility of surgical reintervention, the treatment adopted was a new protocol of conservative management, able of inducing the healing of the fistula [4].

\section{Case Report}

The patient, 75-year-old man with a suspect of Squamous Cell Carcinoma of Tongue (Figure 1) and no methastasis detected with imaging and clinical examination in lymph nodes of the neck, was submitted to the intervention of excision of neoplasia. After follow up with monthly cervical lymph node ultrasonic exam, we notice at 
the second control the presence in submandibular region of lymph nodes with characteristics of malignity. At radiological investigation, CT exam showed the presence of lymph node malignity bilaterally: in relation to diagnostic results bilateral neck dissection was performed. Modified radical neck dissection identified metastasis of squamous carcinoma in cervical lymph nodes level IB, level III on the right and level IIA and IIB on the left.

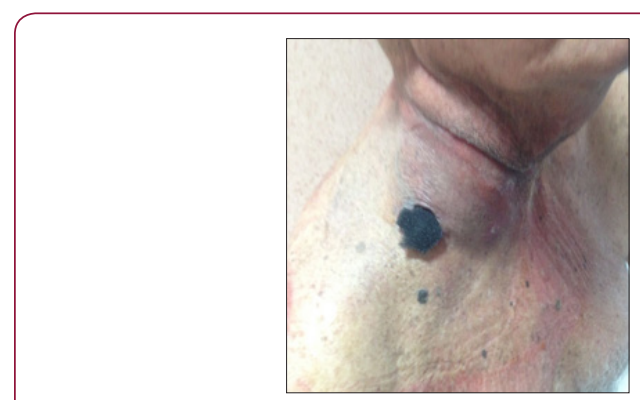

Figure 1.

The patient in I postoperative day resulted in optimal clinical conditions; in right cervical drainages there was the presence of 40 $\mathrm{ml}$ of sieroematic liquid and on left side there was $50 \mathrm{ml}$ of liquid with the same characteristics; in II post-operative day the patient resumed a free diet. After drainages removal, swelling in neck region appears (Figures $2 \& 3$ ) $150 \mathrm{ml}$ of sieroematic liquid on the left and $170 \mathrm{ml}$ of liquid serum-milky liquid on the right were gathered. After drainages reintroduction, supraclavicoular compressive medication was made and it was administered intravenous antibiotic therapy and rehydration. The following day the amount of serum-milky liquid tripled: in particular from the right drainage was about $450 \mathrm{ml}$. The following day (IV post-operative day) the right cervical output drainage was hardly reduced (about 300 $\mathrm{ml}$ of serum-milky liquid ) in contrast with bare secretion in left drainage. The microscopic examination of the milky fluid showed the absence of cancer cells, but revealed copious inflammatory elements , mainly white blood cells , rare red cells and emulsified fats.

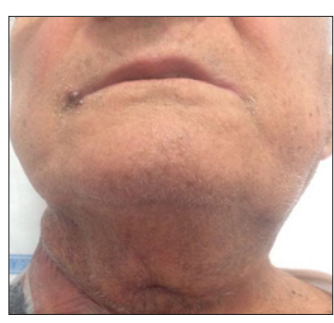

Figure 2.

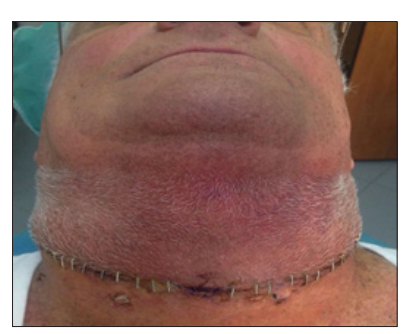

Figure 3.
In the following days was notice another increase of amount of liquid secreted by right cervical drainage, 1050cc of serum-milky liquid, so in the clinical suspicion of a chylous fistula according with previous experience 1, enteral nutrition was interrupted and began a total parenteral nutrition therapy (TPN $2000 \mathrm{kcal} /$ day) 2. Complementary of a TPN, Octreotide continuous iv infusion was made, but was not reported any significant benefit of clinical conditions: in XI post operative day, Negative Pressure Therapy 3 was performed . Until the XIV day after the operation the patient continued with Negative Pressure Therapy and antibiotics; from cervical drainage there were collected $830-850 \mathrm{ml}$ of serous liquid / day. General condition of the patient could not support a surgery event: so conservative management was adopted. Indication of new protocol, based on sclerosant therapy performed twice a day, was made with the use of atossisclerol 2\%, Rifocin 500mg diluted with $100 \mathrm{ml}$ of physiologic solution, injected through cervical drainage clamped after injection for 20 subsequently minutes. The days after the procedure was going on a constant reduction of secretion with surgical hearing after 6 days of this procedure, confirmed by clinical (Figures 4 \& 5) and imaging exams.
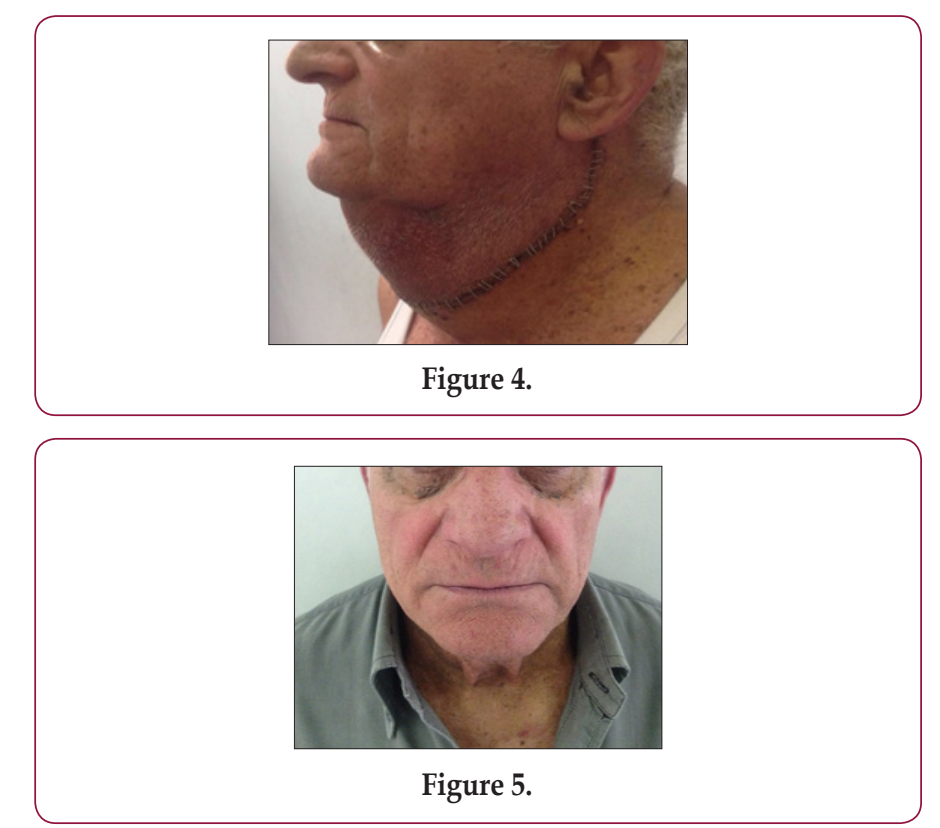

\section{Discussion}

The various possibilities in clinical presentations of such challenging lymphatic duct injuries require an appropriate multidisciplinary approach. Primary prevention of these complications can be achieved through adequate surgical planning to minimize lesions, including patient-based assessment. In the possibility of this complication, a correct and functionally treatment is required, even referred to the clinical condition of the patient.In literature there is a paucity of procedures in treatment of this injuries following head and neck surgery; in an old debilitated patient, the most appropriate treatment should include mainly conservative than surgical approaches. Nonsurgical options consist of low-fat diet with medium-chain triglycerides, total parenteral nutrition, careful monitoring of fluid and electrolytes, drainage of the leakage, somatostatin analogs such as octreotide, negativepressure wound therapy and sclerosant therapy. 
The presence of lymph nodal relapse of pathology in the neck that could have determinated infiltration and compression of lymphatic structures as common trunk of lymphatic duct should be considerated in the localization of surgery reinterventation [4] . In the majority of the surgical procedures, after local toilette there is application of drainage with small aspiration and compressive medication. In several patients the utilization of adjuvant therapy could limit the opening of lymphatic shunts implicating the appearance of fistula even if with low output. Otherwise in a small number of patient, chylous fistula is not to be considered iatrogenous or rather surgically determinated, because wound fibrotic phenomena could condition a limitated opening of vicariant shunts.The volume and the characters of outflow material from the fistula indicate the kind of conservative treatment [5-6].

The prevention of complication in postoperatory period and the eventual malnutrition is represented by reintegration proteic and idroelectrolytic riequilbration [7]. Where is supposed a damage of lymphatic duct and it is possible to individuate two moncons, surgical ligature of them is indicated [8], even if in the majority of the cases is decisive a medical treatment with analogous of somatostatine [9-11]. Despite the technological advances and new approaches described in the recent literature, there is still no clear treatment algorithm for chylous fistula. Functional repair of the thoracic duct injury should be the preferred solution, rather than an approach that obliterates the thoracic duct or lymphatic, chylous pathways, as this can have unwanted consequences such as redistribution of flow to produce distal complications. In case of contraindications of surgical approach, conservative treatment represent a therapeutic rational choice.

Therefore a clear set of standard treatment guidelines is still lacking, although there is a plethora of conservative treatment approaches described in the literature. In our case, clinical conditions of the patient don't allow to proceed with surgical treatment, so a conservative non surgical approach was chosen. The rationale for nutritional management includes decreasing the production and flow of lymph and chyle, replenishing fluid and electrolytes losses, and preventing malnutrition. Options for nutritional care are a low-fat diet (LFD) or fat-free diet (FFD), enteral nutrition with a specialized formula, parenteral support without oral intake, or some combination of the above. In our patient, even he was well nourished and can tolerate oral intake, a LFD or, possibly, a completely FFD wasn't choosen as an option :it is nearly impossible to remove all fats from the diet. Nutritional status was monitored carefully: fatsoluble vitamins together with essential fatty acids (EFAs), multivitamins, and minerals was added to ensure complete nutrient intake on this restrictive regimen.

Our patient required supplemental nutritional support, such as infusion of a TPN fat-free specialized formula. Medium-chain triglycerides (MCTs) have the advantage of being transported directly to the liver through the portal vein, rather than by the lymphatic system, and for this reason are often recommended. Otherwise it is an evidence that lymph fluid contained a significant amount of medium-chain fatty acids, particularly on a very high
MCT diet. In literature is described how authors have reservations about opting for an MCT diet over total parenteral nutrition (TPN) : triglyceride and chylomicron levels can rise on an MCT and wateronly diet, and increase output drainage by $20 \%$ in some patients, recommending to use TPN in the first instance, followed by MCT when the drainage is significantly reduced. From XIX day after surgery, after resolution of milky serum output through drainage, enteral nutrition with a specialized formula was considered [12]. Options for enteral nutrition include MCT-based formulas, very low fat elemental formulas, or a modified regimen using a fat-free oral supplement. Recent literature suggests that enteral nutrition may be effective if chyle output is less than $1 \mathrm{l} /$ day.

Specifically, a lowfat, semi-elemental formula may be adopted if output is less than $0.5 \mathrm{l} /$ day. In contrast, if output is more than 0.5 l/day, an elemental formula may be necessary. There are no trials or data to guide the selection of formula, although clinicians should evaluate the specific formula to be used to determine if it meets full EFA needs, as well as full vitamin, mineral, and micronutrient needs for each individual patient.

Our way of nutritional treatment is conformed with clinical evidence described in literature: TPN is generally recommended for outputs exceeding $1 \mathrm{l}$ /day and in a trial of TPN vs LFD, the former was more effective in closing the chyle leak [13]. However, there may be risks of increased infection and venous thrombosis with continued TPN use [14]. If patients are not responding, one advantage of TPN with intravenous lipid emulsions (IVLEs) is that it is delivered directly into the blood stream, bypassing the lymphatic system, and thus does not contribute to chyle flow. On occasion, IVLE may also be a valuable addition to LFD, FFD, or enteral regimens because they provide a source of calories, as well as EFA.Complementary of a TPN , could be adequate the use of somatostatin. Somatostatin and its analog octreotide act on both endocrine and paracrine pathways by reducing chyle production, inhibiting pancreatic secretion and gastrointestinal secretion, decreasing hepatic venous pressure, and reducing splanchnic blood flow [15].

Octreotide has been used in the treatment as it acts directly on vascular somatostatin receptors to minimize the excretion of lymphatic fluid [16-17]. Negative-pressure wound therapy (NPWT), first introduced in 1997, is a relatively new treatment for complex, poor healing wounds [18]. However, there are few published studies on the applicability of NPWT for head and neck wounds [19-20]. Even if in literature is described the successful use of NPWT for a postoperative chylous fistula after neck dissection using a relatively low pressure of $50 \mathrm{mmHg}$ to avoid an unwanted increase in drainage because of the negative pressure, in our case a NPWT with a pressure of $125 \mathrm{mmhg}$ doesn't resolve the lymphatic outflow even through we found that the amount of drainage decreased after the first day of NPWT without fistula closing within 4 days. It is likely that this early closure was also because of wound shrinkage and removal of excessive fluid around the fistula by NPWT. It is important to note that massive bleeding occurred when NPWT has been applied around major vessels [21]. 
Chylous fistulas are generally located next to the internal jugular vein in the lower neck. Therefore, it is essential to avoid rupture of his and the common carotid artery when NPWT is used in the head and neck region, this complication could be avoided by using gauze to cover the tip of the tube connected to the NPWT device and the soft tissues encompassing the major vessels. NPWT appears to be a promising treatment for chylous fistula with low levels of chyle output [22]. However, the efficacy and safety for chylous fistulas with high outputs, which would drain more than 1 l/day, have yet to be established : in our case, this was not successfully. Despite this reservation, NPWT is likely to become a promising treatment for chylous fistulas after neck dissection, as it is minimally invasive, less burdensome to patients, and as in our case it can be applied when other conservative treatments fail.Our new, unique conservative approach - TPN, octreotide associated to empiric procedure of sclerosant treatment- determinate surgical hearing of the chylous flow after 6 days of introduction of sclerosant solution's washing twice a day : atossisclerol $2 \%$, Rifocin 500mg diluted with $100 \mathrm{ml}$ of physiologic solution, injected through cervical drainage clamped after injection for 20 subsequently minutes. The injection of this sclerosant agent into supraclavicular wound bed through the drainage tube determined rapid decline in fistula output and the solution of this complication after 6 days .

\section{Conclusion}

Because of the anatomical complexities, such as proximity to major vessels and variations, lymphatic duct injuries may be very challenging to manage in neck dissection, which carry potentially serious consequences if not promptly identified and treated appropriately. Despite the technological advances and new approaches described in the recent literature, there is still no clear treatment algorithm for chylous fistula. Functional repair of the thoracic duct injury should be the preferred solution, rather than an approach that obliterates the thoracic duct or lymphatic pathways, as this can have unwanted consequences such as redistribution of flow to produce distal complications. In case of contraindications of surgical approach, conservative treatment represent a therapeutic rational choice: our unique conservative approach could be consider as a new protocol in resolution of chylous fistula.

\section{References}

1. De Gier HH, Balm AJ, Bruning PF, Gregor RT (1996) Systematic approach to the treatment of chylous leakage after neck dissection. Head Neck 18(4): 347-351.

2. Masud MK, Ahmad SM, Karim MA, Ferdouse F, Fakir AY (2014) Complications of neck dissection at a tertiary level hospital: study of 30 cases. Mymensingh Med J 23(4): 658-666.

3. Smoke A, Delegge MH (2008) Chyle leaks: Consensus on management? Nutr Clin Pract 23(5): 529-532.

4. Dhiwakar M, Nambi GI, Ramanikanth TV (2014) Drain removal and aspiration to treat low output chylous fistula. Eur Arch Otorhinolaryngol 271(3): 561-565
5. Kannan RR, Mahajan V, Ayyappan S (2001) Management of chyle fistulae following surgery in the neck. Indian J Cancer 38(2-4): 117-120.

6. Barth U, Wasseroth K, Rahms V, Albrecht R, Meyer F (2016) Chylous complications of various severity and manifestations within diverse compartments in reconstructive vascular surgery. Pol Przegl Chir X 90(3): 43-48.

7. Polistena A, Vannucci J, Monacelli M, Lucchini R, Sanguinetti A (2016) Thoracic duct lesions in thyroid surgery: An update on diagnosis, treatment and prevention based on a cohort study. Int J Surg 28(Suppl 1): S33-S37.

8. Campisi CC, Boccardo F, Piazza C, Campisi C (2013) Evolution of chylous fistula management after neck dissection. Curr Opin Otolaryngol Head Neck Surg 21(2): 150-156.

9. Suver DW, Perkins JA, Manning SC (2004) Somatostatin treatment of massive lynphorrhea following excision of lymphatic malformation. Int J Ped Otolaryngol 68(6): 845-850.

10. Valentine CN, Barresi R, Prinz RA (2002) Somatostatin analog treatment of a cervical thoracic duct fistula. Head Neck 24(8): 810-813.

11. Priego Jiménez P, Collado Guirao MV, Rojo Blanco R, Grajal Marino R, Rodríguez Velasco G et al. (2008) Chyle fistula in right cervical area after thyroid surgery. Clin Transl Oncol 10(9): 593-596.

12. Spiro JD, Spiro RH, Strong EW (1990) The management of chyle fistula. Laryngoscope 100(7): 771-774

13. Ramos W, Faintuch J (1986) Nutritional management of thoracic duct fistulas: a comparative study of parenteral versus enteral nutrition. J Parenter Enteral Nutr 10(5): 519-521.

14. Rimensberger PC, Muller-Schenker B, Kalangos A (1998) Treatment of a persistent postoperative chylothorax with somatostatin. Ann Thorac Surg 66(1): 253-254.

15. Barili F, Polvani G, Topkara VK (2007) Administration of octreotide for management of postoperative high-flow chylothorax. Ann Vasc Surg 21(1): 90-92.

16. Jain A, Singh SN, Singhal P, Sharma MP (2015) A prospective study on the role of octreotide in management of chyle fistula neck. Laryngoscope 125(7): 1624-1627.

17. Sharkey AJ, Rao JN (2012) The successful use of octreotide in the treatment of traumatic chylothorax. Tex Heart Inst J 39(3): 428-430.

18. Chang F, Cheng D, Qian M, Lu W, Li H, et al. (2016) Thoracic Duct Chylous Fistula Following Severe Electric Injury Combined with Sulfuric Acid Burns: A Case Report. Am J Case Rep 17: 730-733.

19. Andrews BT, Smith RB, Hoffman HT (2008) Orocutaneous and pharyngocutaneous fistula closure using a vacuum-assisted closure system. Ann Otol Rhinol Laryngo 117(4): 298-302.

20. Dhir K, Reino AJ, Lipana J (2009) Vacuum-assisted closure therapy in the management of head and neck wounds. Laryngoscope 119(1): 54-61.

21. Orgill DP, Bayer LR (2011) Update on negative-pressure wound therapy. Plast Reconstr Surg 127 (Suppl. 1): 105S-115S.

22. Kadota H, Kakiuchi Y, Yoshida T (2012) Management of chylous fistula after neck dissection using negative-pressure wound therapy: a preliminary report. Laryngoscope 122(5): 997-999. 
ISSN: 2574-1241

DOI: 10.26717/BJSTR.2018.09.001853

Raffaele Rauso. Biomed J Sci \& Tech Res

CC) This work is licensed under Creative

Submission Link: https://biomedres.us/submit-manuscript.php

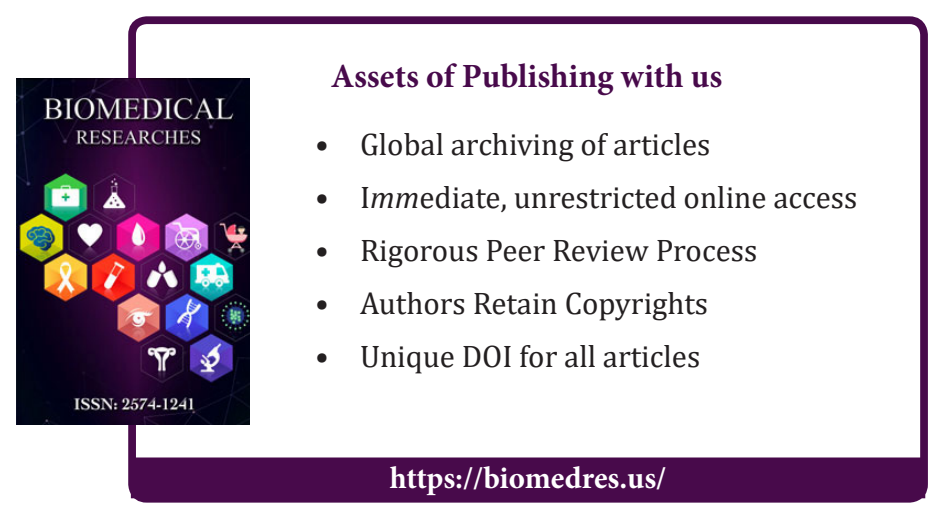

